\title{
Review on Omega-3-Supplementation in Eating Disorders
}

Yazed Mohammed Alashmali ${ }^{1}$, Abdulrahman Sameer Basabrain ${ }^{1}$, Abdulaziz Ibrahim Habadi ${ }^{2}$, Noura Ghaleb Alroweli ${ }^{3}$, Hamza Ahmed Bana ${ }^{4}$, Khaled Farouk Helmi Faisal ${ }^{5}$, Maryam Abdalhadi Altayeb $^{6}$, Luay Abdullah Alqurashi ${ }^{2}$, Sabei, Ali Ibrahim H. ${ }^{7}$, Elyas Mahmoud Alsa'ati ${ }^{2}$

1- Royal College of Surgeons, Ireland, 2- Department of Psychiatry, Al-Amal Hospital, Ministry of Health, Jeddah, Saudi Arabia,3- Ministry of Health, Jeddah, Saudi Arabia, 4- Faculty of Medicine, King Abdulaziz University, 5- Imam Abdulrahman Alfaisal Hospital (Riyadh),6- Batterjee Medical College,7 -Salhaba and New Sabya Primary Health Care Centre in Jazan

\begin{abstract}
Background: Eating disorders are complex, potentially life-threatening conditions, characterised by disturbances in eating behaviours and extreme fear of weight gain with significant morbidity and mortality rates. Individuals avoid fattening foods. This avoidance of fattening foods may lead to the avoidance of foods which may include those with omega 3 fatty acids. Aim of the work: Aim of the review is to explore the consequence of starvation on the brain both in adolescence and in adults and particularly due to omega 3 deprivation. It also aims to explore the role of the benefits and possible mechanisms of omega 3 supplementation on other mental health conditions and to consider these implications for eating disorders. Methods: The search was undertaken by using PubMed and the search terms, eating disorders, anorexia nervosa and Omega-3. Alternatively, search terms such as depression, schizophrenia or other mental health conditions and omega-3 were used. References lists of identified reviews and articles were also searched for relevant empirical studies. Results: According to a growing body of evidence, the role and importance of omega-3 fatty acids in mental health is well-recognised and well-established to be beneficial. Fatty acids are fundamental components of the brain and are necessary for normal body physiology. Since the body cannot synthesize these fats, it has to be completely obtained from the diet. Brain tissue is rich in omega-3 fatty acids. These acids have a major role within cell membranes and in connections between neurons.Omega-3 FAs deprivation may be correlated with the happening of many psychiatric conditions, such as obsessive-compulsive disorder, depression, bipolar disorder, and schizophrenia. Conclusion: The clinical research evidence on omega-3 supplements in eating disorders is preliminary and not definitive in comparison to other mental health disorders. Thus, this will be an important area for future research, and may lead to the development of newer interventions.
\end{abstract} Keywords: Omega-3 supplements, eating disorders, preliminary, mental health disorders.

\section{INTRODUCTION}

\section{Eating disorders and fat deprivation}

Eating disorders are serious psychological illnesses marked by disturbances in eating behaviour and extreme fear of weight gain. They have a core psychopathology centred on food, eating habits and• body image concerns. These habits eventually lead to the deterioration of the individual's physical and mental health ${ }^{[1,2]}$. Eating disorders have one of the highest death rates of all psychiatric diagnoses. The number of deaths in those with anorexia nervosa was 11.6 times• higher in comparison with their healthy counterparts of the same age and sex. In those with bulimia, the rate is 1.3 times ${ }^{[3]}$. Due to the core psychopathology and the cognitive distortion associated with these disorders, individuals restrict their dietary intake in order to prevent weight gain. This self-starvation may lead to. serious nutritional deficiencies. The impact of nutritional self-deprivation ranged from general poor health to life-threatening conditions and could have huge consequences on the body and mind. Patients with anorexia have the tendency to deprive fat during their self-induced starvation due to the fat phobic nature of this disorder. Thus, these patients are more likely to develop severe omega-3 deficiency which may have serious impact on the brain ${ }^{[4]}$.

\section{Omega-3 Fatty Acids}

Omega-3 fatty acids are polyunsaturated fatty acids that are essential for human health and development. There are three types involved in human physiology, namely: Alpha-Linolenic acid. (ALA): it is an 18 carbon chain fatty acid with 3 double bonds. It is known as dietary essential FAs, so called because it cannot be synthesised de novo and it must be acquired from the diet. Dietary resources includes walnut and flaxseed oil.

Eicosapentaenoic acid. (EPA): It is a 20 carbon chain fatty acid with 5 double bonds. It could be acquired through the conversion of ALA within the liver cells. However, the conversion capacity in human is very limited and inefficient. Thereby, it's mainly acquired from dietary sources such as fish oil

Docosahexaenoic acid. (DHA): It is a 22 carbon chain fatty acid with 6 double bonds. It is the most abundant fatty acid in the brain which makes up an average of $10 \%$ of fat in our brains. Its acquisition is similar to EPA in that it could be synthesised from ALA inefficiently but the main source would be marine oils ${ }^{[5,6]}$.

\section{Role of Omega-3 in the Brain}

These types of lipids play a key part in the body growth, development and metabolism as they are involved in cell signalling and constitute an integral part of cell 
membranes throughout the body especially the brain. From these fatty acids many bioactive-metabolites are produced. These metabolites have anti-inflammatory properties such as docosanoids from DHA and EPAderived eicosanoids; collectively known as specialized pro-resolving mediators ${ }^{[7]}$. DHA of the omega-3 fatty• acids is the most abundant lipids in the brain and it's crucial in maintaining baseline regulatory functions. The reason why it is crucial is that DHA comprises about $50 \%$ of the neurons' plasma membranes. Drops in DHA levels have been associated with cognitive decline and many other mental health problems ${ }^{[8]}$. One of the proposed mechanisms of action by which DHA and EPA help maintain the integrity of the brain function is by reducing the inflammatory reactions and protecting the brain tissue against damage. This mechanism may be achieved by DHA itself and DHA-• derived docosanoids as they have anti-inflammatory properties and neuro-protective function ${ }^{[9,10]}$

\section{METHODS}

A comprehensive literature review was conducted to systematically identify all the studies that were potentially relevant. Firstly, the literature review focussed on articles studying the impact of omega-3 supplements on various mental health conditions. Then, it focused on articles studying the impact of omega-3 supplements on eating disorders particularly anorexia nervosa.

The search was undertaken by using PubMed and the search terms, eating disorders, anorexia nervosa and. Omega-3. Alternatively, search terms such as depression, schizophrenia or other mental health conditions and omega-3 were used. References lists of identified reviews and articles were also searched for relevant empirical studies. Additionally, Google Scholar was used. It offered a wider coverage and led to different literature that weren't available in PubMed. Advance search on Scholar was used as appropriate to achieve more specific results and to reduce the results to a more manageable number. Ninety-eight papers were generated and inspected for relevance and quality. of which, fifty-five papers were included. Inclusion criteria were based on the relevance of these trials to the topic of this paper. In addition, quality measures such as: trials being randomised double-blinded and placebo controlled have been taken into considerations. A series of drafts were then prepared and refined leading to the final draft. The final draft was then reviewed by Dr Sarah Prasad, a consultant psychiatrist in St. Patricks University Hospital.

\section{RESULTS}

\section{Role of Omega-3 in Mental Health}

According to a growing body of evidence, the role and importance of omega-3 fatty acids in mental health is well-recognised and well-established to be beneficial. The scientific-clinical trials have provided sufficient evidence about the link between omega-3 deficiency and various mental health disorders. Such evidence was established in the following disorders:

\section{Depression}

Many studies have suggested that consuming sufficient amount of omega-3 FAs will provide a powerful protection against depression. For example, a large Norwegian study of nearly 22,000 participants revealed that the longer the participants took cod liver oil, the less likely they were to have high levels of depression ${ }^{[11]}$. According to other studies, omega-3 supplements can also enhance the mood in those who already suffer from depression ${ }^{[12]}$.

\section{Schizophrenia}

There was a considerable evidence to suggest that omega-3 FAs supplements may be an effective adjunct to anti-psychotic therapy for managing schizophrenic patients. Most studies have focussed on the EPA fatty acid and have demonstrated clinical improvement in the vast majority of patients. On the other hand, some studies have shown a preventive effect of omega-3 against schizophrenia in highly susceptible individuals. This was done by examining individuals who have a strong family history of schizophrenia or had shown early signs of schizophrenia such as delusions or hallucinations ${ }^{[13,14]}$.

\section{Bipolar Disorders}

Although many studies have demonstrated the effect of omega-3 fatty acid in improving the depressive symptoms in bipolar patients, there is still no evidence, that it has any effect on attenuating the maniac symptoms ${ }^{[15]}$.

\section{Attention Deficit Hyper-activity Disorder}

From meta-analysis of omega-3 supplementation trials, there was some evidences that omega-3 supplements could create modest improvement in ADHD symptoms. So, it could be part of the established therapy as well have also shown that omega-3 levels are reduced in children with ADHD ${ }^{[16]}$.

\section{Other Psychiatric Disorders}

Smaller clinical trials on omega-3 supplementation have been conducted on other mental health disorders such as borderline personality disorder, obsessivecompulsive disorder and Alzheimer's disease. Results from these trials were often mixed ,but they were usually in favour of omega-3 supplements as preventive and therapeutic tools ${ }^{[17,18]}$.

\section{The Impact of Starvation}

Brain tissue is rich in omega- 3 fatty acids. These acids have a major role within cell membranes and in 
connections between neurons. DHA and EPA in particular are major structural components of neuronal membranes, and any change in the fatty acid composition of neuronal membranes can lead to functional changes in the activity of receptors and other proteins embedded in the membrane phospholipid and thus canresult in neuro-inflammation ${ }^{[19]}$. Therefore, any decrease in concentration of these fats can lead to neuronal membrane deformity and thus, neuronal inflammation, serotonin depletion, decreased energy metabolism in the neurons and eventually neuronal death ${ }^{[20]}$. Omega-3 FAs deprivation may be correlated with the happening of many psychiatric conditions, such as obsessive-compulsive disorder, depression, bipolar disorder, and schizophrenia. It has been also argued that deformities in the membrane phospholipid are present in patients with mental health disorders ${ }^{[21]}$. Exploration of the mechanism of action of omega-3 in these disorders may help establish newer protocols that feature omega-3 supplements as an adjunctive therapy in the treatment of other mental health conditions such as eating disorders.

\section{DISCUSSION}

\section{Mode of Action and Pathophysiology}

As it widely accepted, the brain neurotransmitter serotonin has a huge influence on cognitive functions and behaviours including social behaviour, decisionmaking, mood and impulsive behaviour. Serotonin depletion is a unifying feature of many clinical mental disorders such as attention deficit hyperactivity disorder (ADHD), bipolar disorder, schizophrenia and depression ${ }^{[22]}$. Continues inflammation in the brain tissue may be the key to serotonin depletion and the development of mental disorders. This is because inflammation results in neuronal malfunction which lead to various neurotransmitters depletion which will eventually lead to neuronal death ${ }^{[23]}$. By giving omega3 -supplements to patients with mental health disorders, we are aiming to restore the balanced structure of the neuronal membrane phospholipid and thus prevent further inflammation. This is achieved by the bio-active derivatives of both DHA and EPA fatty acids which act as inflammatory signalling molecule inhibitors and thus can result in maintenance of neurotransmitters level ${ }^{[24]}$. Consumption of omega-3-supplements can maintain a healthy and balanced structure of the neuronal membrane phospholipid. This will aid in neuronal functioning and will subsequently prevent neuroinflammation which may negatively impact serotonin levels in the brain.

\section{Omega-3-Supplements and Eating Disorders}

As in other psychiatric disorders, it is thought that malfunction of serotonin pathways in the limbic system and prefrontal cortex and thus depletion of serotonin is evident in eating disorder patients. In addition, few studies with anorexia nervosa patients revealed that these patients had a change in the phospholipid membrane composition in addition to a decreased level of omega-3 fatty acids ${ }^{[25]}$. Recent studies have also demonstrated that the lack of omega- 3 fatty acids modulate changes in the level of anorexigenic neuropeptides in the brain. These neuropeptides reduce appetite and food intake, and, as a result, causes weight loss to occur ${ }^{[26]}$. Therefore, it's predicted that omega-3supplements can work in the direction of repairing these brain abnormalities i.e. act in the same way as in depressive disorders, as an anti-inflammatory agents, preventing neuronal damage by stabilizing and enhancing the neuronal membrane integrity and function, and by restoring nerves conduction throughout the serotonin affected pathways. Also, they would have a role in stimulating the production and release of orexigenic neurotransmitters such as neuropeptide $\mathrm{Y}$ which are responsible for stimulating the appetite and increase food intake ${ }^{[26,27]}$.Furthermore, we can argue that omega-3-supplements can be used as a treatment for the other clinical manifestations of eating disorders such as ventricular arrhythmias, osteoporosis and sudden death syndrome. All of the previous have been shown in research and trials to be reduced in incidence upon the consumption of omega-3 supplements ${ }^{[27]}$. Other mental health problems are also common in patients with eating disorders especially depression, bipolar and borderline personality disorders. The role of omega-3-supplements in these disorders has also been established to be beneficial.

\section{CONCLUSIONS AND RECOMMENDATIONS}

Most scientific literatures suggested that omega-3 fatty acids act on many mechanisms involved in the physiopathology of mental illnesses. However, it is impossible, at the moment, to establish clinical evidence on their role in anorexia nervosa. This is due to the little body of research studying the link between omega-3-supplements and the symptoms and consequences of eating disorders. So far, only limited number of clinical trials has explored the therapeutic effect of omega-3-supplements on eating disorders and these trials have given mixed and conflicted results. That's probably due to the lack of control group in some of these trials and the very low number of participants in others. Because the previous and the ongoing work done with omega-3 fatty acids lacked standardization of therapeutic doses and were generally conducted with small samples and no control groups, further clinical trials are necessary to establish the evidence for the therapeutic potentials of these supplements. In order for these trials to be viable and produce accurate results, they need to be large, randomised, double blinded, standardised and more complex placebo-controlled. In summary, the clinical research evidence on omega-3 
supplements in eating disorders is preliminary and not definitive in comparison to other mental health disorders. Thus, this will be an important area for future research, and may lead to the development of newer interventions.

\section{REFERENCES}

1. Herpertz S, Hagenah U, Vocks S et al. (2011): The diagnosis and treatment of eating disorders. Dtsch Arztebl Int., 108(40):678-685. A

2. Bell C, Bulik C, Clayton $P$ et al. (2000):Practice guideline for the treatment of patients with eating disorders (revision). American Journal of Psychiatry, 157(1):1-39.

3. Hudson JI, Hiripi E, Pope HG et al. (2007): The Prevalence and Correlates of Eating Disorders in the National Comorbidity Survey Replication. Biol Psychiatry,61(3):348-58.

4. Keel PK, Dorer DJ, Eddy KT et al. (2003): Predictors of mortality in eating disorders. Arch Gen Psychiatry,60(2):179-83.

5. Lee $\mathbf{S}$ (2004): Fat phobia in anorexia nervosa: Whose obsession is it? Eating Disorders and Cultures in Transition Annu Rev Nutr.,21: 37-50.

6. Scorletti E and Byrne CD (2013): Omega-3 fatty acids, hepatic lipid metabolism, and nonalcoholic fatty liver disease. Annu Rev Nutr., 33:231-48.

7. Burdge GC and Calder PC (2005): Conversion of $\alpha$ linolenic acid to longer-chain polyunsaturated fatty acids in human adults. Reprod Nutr Dev., 45:581-597.

8. Orr SK, Palumbo S, Bosetti F et al. (2013): Unesterified docosahexaenoic acid is protective in neuroinflammation. J Neurochem.,127(3):378-93.

9. Gleissman H, Johnsen JI and Kogner P (2010): Omega-3 fatty acids in cancer, the protectors of good and the killers of evil? Experimental Cell Research, 10:1365-1373.

10. Orr SK and Bazinet RP (2008): The emerging role of docosahexaenoic acid in neuroinflammation. Curr Opin Investig Drugs,9(7):735-43.

11. Ozsoy O, Seval-Celik Y, Hacioglu G et al. (2011): The influence and the mechanism of docosahexaenoic acid on a mouse model of Parkinson's disease. Neurochem Int.,59(5):664-670.

12. Raeder MB, Steen VM, Vollset SE et al. (2007): Associations between cod liver oil use and symptoms of depression: The Hordaland Health Study. J Affect Disord., 101(1-3):245-249.

13. Hallahan B, Hibbeln JR, Davis JM et al. (2007): Omega-3 fatty acid supplementation in patients with recurrent self-harm. Single-centre double-blind randomised controlled trial. Br J Psychiatry, 190:118122.
14. Emsley R, Oosthuizen P, van Rensburg SJ (2003): Clinical potential of omega-3 fatty acids in the treatment of schizophrenia. CNS Drugs, 17(15):1081-1091.

15. Amminger GP, Schäfer MR, Papageorgiou K et al. (2010): Long-chain omega-3 fatty acids for indicated prevention of psychotic disorders: a randomized, placebo-controlled trial. Arch Gen Psychiatry, 67(2):146-54.

16. Sarris J, Mischoulon D, Schweitzer I (2012): Omega3 for bipolar disorder: Meta-analyses of use in mania and bipolar depression. J Clin Psychiatry,73(1):81-6.

17. Bloch MH, Qawasmi A (2011): Omega-3 fatty acid supplementation for the treatment of children with attention-deficit/hyperactivity disorder symptomatology: Systematic review and meta-analysis. Journal of the American Academy of Child and Adolescent Psychiatry, 50(10):991-1000.

18. Peet $M$ and Stokes $\mathbf{C}$ (2005): Omega-3 fatty acids in the treatment of psychiatric disorders. Drugs, 65(8):1051-9.

19. Freeman MP, Hibbeln JR, Wisner KL et al. (2006):Omega-3 fatty acids: Evidence basis for treatment and future research in psychiatry. Journal of Clinical Psychiatry,68(2):338-344.

20. Calder PC (2011): Fatty acids and inflammation: The cutting edge between food and pharma. European Journal of Pharmacology. Eur J Pharmacol.,668:50-58.

21. Conklin SM, Harris JI and Manuck SB (2007): Serum ??-3 fatty acids are associated with variation in mood, personality and behavior in hypercholesterolemic community volunteers. Psychiatry Res.,152(1):1-10.

22. Berger M, Gray JA and Roth BL (2009): The expanded biology of serotonin. Annu Rev Med., 60:35566.

23. Mythri RB, Venkateshappa C, Harish G et al. (2011): Evaluation of Markers of oxidative stress, antioxidant function and astrocytic proliferation in the striatum and frontal cortex of Parkinson's disease brains. Neurochem Res., 36(8):1452-63.

24. Dyall SC, Michael GJ, Michael-Titus AT (2010): Omega-3 fatty acids reverse age-related decreases in nuclear receptors and increase neurogenesis in old rats. $\mathbf{J}$ Neurosci Res., 88(10):2091-102.

25. Prior PL and Galduróz JC (2012): (N-3) Fatty acids: molecular role and clinical uses in psychiatric disorders. Adv Nutr .,3(3):257-65.

26. Goncalves CG, Ramos EJB, Suzuki S et al. (2005): Omega-3 fatty acids and anorexia. Curr Opin Clin Nutr Metab Care, 8(4):403-7.

27. Wall R, Ross RP, Fitzgerald GF et al. (2010): Fatty acids from fish: The anti-inflammatory potential of longchain omega-3 fatty acids. Nutrition Reviews, 68(5):280-289. 